
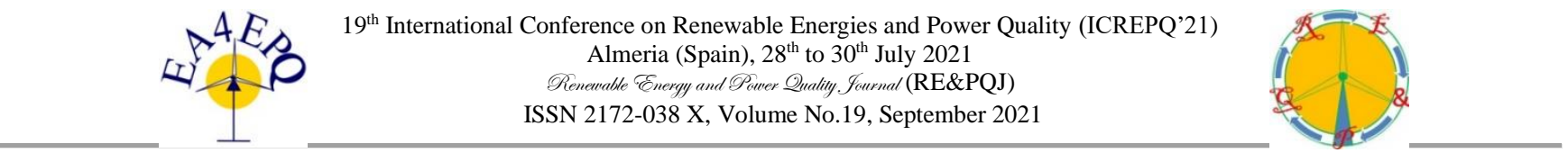

\title{
Airfoil optimization for small horizontal axis wind turbine
}

\author{
Cristhian Leonardo Pabón Rojas ${ }^{1}$, Carlos Andrés Trujillo Suarez ${ }^{1}$, Juan Carlos Serrano Rico² and Elkin \\ Gregorio Flórez Serrano ${ }^{2}$ \\ ${ }^{1}$ Mechanical Engineering Program, Engineering Faculty \\ Universidad de Antioquia \\ Medellín, Colombia \\ Phone:+0057 (4) 2198332,e-mail: cristhian.pabon@udea.edu.co, carlos.trujillo@udea.edu.co
}

${ }^{2}$ Mechanical Engineering Program, Faculty of Engineering and Architecture.

Universidad de Pamplona

Pamplona, Colombia

Phone: +0057 (7) 5685303 e-mail: jcserrano@unipamplona.edu.co

\begin{abstract}
In order to take advantage of the low wind speed found in the Colombian territory, a gradient-based optimization process (GBA) of 2 airfoils is carried out, using the Xfoil software to evaluate the interactions. The shapes chosen will be destined for the root and for the middle zone of a blade for a small horizontal axis wind turbine (sHAWT). The blade will be created from the calculation of the chord and pitch angle with the blade element momentum methodology (BEM) and the SHAWT will be tested by CFD software to check its performance. As a preliminary result, a root-bound airfoil has been obtained with a higher performance than the airfoil used as a bases.
\end{abstract}

Key words: airfoil, optimization, HAWT, lift coefficient, drag coefficient

\section{Introduction}

The increase in population and industrial growth in production demand more and more energy, leading to burning a greater amount of fossil fuels to meet this demand, these fuels generate more pollutants and greenhouse gases. Wind and solar photovoltaic energy are a short and longterm alternative to reduce these pollutants [1]. In Colombia seeks to reduce dependence on traditional energy sources such as fossil fuel sources and large-scale hydropower, the latter represent about $80 \%$ of the energy generated in the interconnected system and periodically is at risk due to natural phenomena such as the El Niño phenomenon that caused an energy crisis in the 90's. In this context, the use of wind energy becomes more attractive due to the generation potential that exists in the country, only in the Guajira region there is a potential of $18 \mathrm{GW}$ although in the rest of the territory it has a great variability of speed with average speeds between 4 and $5 \mathrm{~m} / \mathrm{s}$ can be an exploitable potential [2]. It is clear that the blade assembly constitutes one of the main components of a wind turbine. The wind turbine efficiency depends on the capability of these devices to extract kinetic energy from the wind [3]. there is an opportunity for exploitation that positively impacts the aspects discussed above, taking into account the wind speeds of the Colombian territory, the use of small horizontal axis wind turbines becomes more feasible, but the contribution of some small wind turbines is not viable as indicated [4]. In order to increase the performance of small turbines, the design of low Reynolds number airfoils seems to be a good optimization option, as the information on low Reynolds number airfoils is not yet complete [5]. For that, the blades should be specially designed to transform the force of the wind into the torque needed to generate useful power. Thus, the blade design is focusing onto maximize the rotor power output and to minimize the losses, ensuring higher performance [6]. For low Reynolds numbers, there are boundary layer separation problems because the transition from laminar to turbulent regime does not occur, which decreases the performance of the profiles. This separation bubble, which for high Reynolds numbers can cover $2 \%$ of the profile surface, can cover up to $15 \%$ at Reynolds numbers below $3 \times 10^{5}$ [7]. A twodimensional numerical study Santhanakrishnan [8] demonstrated that very large drag forces are required to produce blade rotation for relevant Reynolds, however, that force is reduced when the wings are flexible or porous. Wang [9] found that the reaction torque of wind turbine increases with increasing Reynolds numbers, indicating its better self-starting characteristics, the average tangential forces are higher at the blade tip and root, respectively, and this improvement increases with increasing Reynolds number. Larger tangential forces on the horizontal blade lead to better power performance.

Despite technological advancements occurred in the last decades, aerodynamic design and assessment are still challenging steps because these machines have to hold up strong and variable operating conditions during their lifetime. The speed of these wind turbines can easily reach Mach numbers in the range of $0.1-0.3$, (being 0.3 the conventional limit) under normal operating conditions. The air flow is assumed to be incompressible, which is reasonable at the usually prevailing Mach numbers in HAWT applications. The Navier-Stokes equations along 
with the continuity equation are solved computationally. [10], as compressibility effects aren't expected to arise can affect the flow field as well as the performance of the wind turbines.

In the study of Menegozzo [11] the $\mathrm{k}-\omega$ SST turbulence model has been adopted to relate the Reynolds stresses to the mean flow. In the same way, Benim [12] assume the flow to be two-dimensional (2D) and statistically in the steady state. For a rotating turbine with fixed blades, this setting resembles a blade-to-blade surface at a fixed radial section, where the turbine rotational speed and the wind speed are constant in time, in a moving (rotating) reference frame. In the sense of a quasi-three dimensional procedures, a three-dimensional model can be constructed by an assembly of such blade-to-blade sections. It shall be noted that the resemblance is, however, approximate in so far, that a curved blade-to-blade surface resulting from a radial section is represented by a flat plane in $2 \mathrm{D}$.

This work shows the main objectives taken into account for the development of the work to evaluate the performance of a small horizontal axis wind turbine to be used in the Colombian territory. Subsequently, it is divided into three main parts. In Part I, the methodology, this explains the selection and parameterization of the selected profiles to be optimized later, in this last procedure the evaluation objective is established, which in this case is to maximize the $C_{l} / C_{d}$ ratio, however, the restrictions of the structural requirements must be considered. The results obtained are shown after the methodology, in this part a model of the wind turbine blade is made based on the BEM methodology, with which the chord size and pitch angle will be calculated for each section along the blade. The conclusions are finally summarized in section 5 .

\section{Objective}

The objective of this work is the design of an airfoil using optimization methods based on an airfoil previously selected for its performance at low Reynolds numbers. With the optimal geometry obtained, to evaluate the performance of a small horizontal axis wind turbine at low wind speeds to be used in the Colombian territory.

\section{Methodology}

\subsection{Airfoil selection for low Reynolds numbers}

A comparison of different free airfoils found in literature, airfoils that are or can be used for wind turbines and that have been designed for low Reynolds numbers, will be carried out. For this performance comparison, the lift coefficient $\left(C_{l}\right)$, drag coefficient $\left(C_{d}\right)$ and their ratio $\left(C_{l} / C_{d}\right)$ will be taken into account. To make this comparison in a practical way, the XFOIL software will be used, a software with lower computational requirements than a CFD one, which has also demonstrated to have enough accuracy for the prediction of the $C_{l}$ and $C_{d}$ coefficients. [13]. The selected aerodynamic profile will function as the base or initial geometry for further optimization in order to obtain an optimal geometry more quickly.

\subsection{Parameterization Method}

The method of Bézier curves is a method of polynomial parameterization that allows a constant control of the polygons to make the geometry adjustable. Defined as a set of control points that allows representation of a complete geometry, in a generalized way the method is extended to a series of polynomials of different order according to the number of control points, the order being equal to n-1 control points of each curve. But this method has disadvantages such as the global change of the geometry due to the single movement of a point, even that characteristic is named "one movement, hundreds of movements" [14]. According to [15] Bezier curve is defined by the equation for given control points $P_{i}$ and Bernstein polynomials

$$
P(t)=\sum_{i=0}^{n} P_{i} B_{i}^{n}(t)
$$

The Bézier curves are based on the Bernstein polynomial $B_{i}^{n}$ which is given by:

$$
B_{i}^{n}=\left(\begin{array}{c}
n \\
i
\end{array}\right)(1-t)^{n-i} t^{i}
$$

Where $\mathrm{i}=0,1,2,3 \mathrm{y}\left(\begin{array}{c}n \\ i\end{array}\right)$ is a binomial coefficient which is given by:

$$
\left(\begin{array}{l}
n \\
i
\end{array}\right)=\frac{n !}{i !(n-i) !}
$$

If $n=3$, the Bezier curve consists of writing the following Bernstein polynomial of $n+1=4$ terms which is constructed as follows:

$$
\begin{gathered}
B_{0}^{3}=(1-t)^{3} \\
B_{1}^{3}=3 t(1-t)^{2} \\
B_{2}^{3}=3 t^{2}(1-t) \\
B_{2}^{3}=t^{3}
\end{gathered}
$$

Therefore the Berntein polynomial for a third degree Bézier curve would be:

$$
\begin{array}{r}
P(t)=P_{0}(1-t)^{3}+3 P_{1} t(1-t)^{2} \\
+3 P_{2} t^{2}(1-t)+P_{3} t^{3}
\end{array}
$$

Where $t$ is a parameter between 0 and 1 ..

As expressed in equation ( 8) four coefficients are required to represent the coordinates of the control points of the domain of the curve and from these the range of variation of each one can be established. By moving one of the control points the whole curve will be affected, consequently, it is necessary to designate the initial values 
of the control points starting from a base profile, in this case the SG604X family of profiles selected as base profiles because although they have been designed for Reynolds number values between 200,000 and 500,000 they show great performance for lower Reynolds numbers.

\subsection{Optimization}

The optimization will aim to minimize or maximize the response of the objective function, [16]

$$
\begin{aligned}
& \text { Minimize }(\mathrm{X}) \\
& g_{j} \leq 0 \quad \mathrm{j}=1, \mathrm{M} \\
& h_{k}(X)=0 \quad \mathrm{k}=1, \mathrm{~L} \\
& X_{i}^{L} \leq X_{i} \leq X_{i}^{U} \quad i=1, \mathrm{~N}
\end{aligned}
$$

Where equation ( 9) defines the objective function that depends on the design variables $X$ and equation (10) and (11) are equalities and inequalities used as a constraint and the equation (12) defines the region in which the minimum response should be sought.

The objective is to decrease the airfoil drag coefficient or increase the ratio of lift coefficient to drag coefficient $\left(C_{l} / C_{d}\right)$ as a nonlinear problem for the solution a gradient based algorithm will be used. Taking into account that it is an algorithm that can fall into a local optimal solution, the previous step of selecting an aerodynamic profile geometry for low Reynolds numbers is carried out.

For the Gradient method used, the variable $X_{0}$ represents the initial position and $X_{1}$ the final position of the first iteration.

$$
X_{1}=X_{0}+a * S_{1}
$$

Where $S_{1}$ is the search direction in the first iteration and $a^{*}$ is the optimal amplitude of motion along the direction $S_{1}$. The optimization process comprises the iteration of this process.

A gradient-based algorithm (GBA) can be more sensitive by using an initial solution as a starting point, in this case a preselected geometry that refines the solution, searching in a smaller space and obtaining an optimal geometry more quickly, so as not to fall into a local solution, the procedure must be repeated several times [17].

\section{Objective evaluation}

The objective is to maximize the relationship $\frac{C_{l}}{C_{d}}$, For this, an interesting and very common option is not to allow the lift coefficient to decrease from the original value and to minimize the drag coefficient. So the objective would be: Minimize the drag coefficient.

\section{Restrictions:}

$>$ The maximum thickness of the profile must be controlled in order not to obtain as a result a very thin profile, in this case comparing it with the thickness of the base family and not allowing it to be much less seems adequate considering that the structural analysis of the blade is based on stresses obtained from work followed by aerodynamic design.

The trailing edge radius must be taken into account since it is simplified to be represented as a point, but in reality, due to the manufacturing process, a minimum radius equal to $0.25 \%$ of the chord value of the profile (chord) is necessary.

\section{Parameters to be known from the evaluation environment}

These parameters, although not directly linked to the optimization problem, are the environment to which the airfoil will be exposed, and must be known beforehand as a prerequisite for software evaluation.

$$
\begin{aligned}
& >\text { Angle of attack }(A o A) \\
& >\text { Reynolds number }(R e) \\
& >\text { Tip speed ratio }(T S R) \\
& >\text { Mach bumber }(M a c h)
\end{aligned}
$$

The Reynolds number is considered as a parameter to be entered in the XFoil software where the performance of the airfoils is evaluated.Equation ( 14) is used to calculate the Reynolds number.

$$
R e=\frac{\rho V c}{\mu}
$$

Where $\rho$ is the air density $\left[\mathrm{kg} / \mathrm{m}^{3}\right], V$ the fluid velocity with respect to the object $[\mathrm{m} / \mathrm{s}]$ in this case airfoil, $c$ the chord or distance between the leading edge and the trailing edge of the airfoil $[\mathrm{m}]$ and $\mu$ the dynamic viscosity of the air $[\mathrm{kg} / \mathrm{ms}]$. The fluid velocity varies, increasing with the radial distance from the center of rotation for each blade section as it is governed by equation (15).

$$
V=\sqrt{u^{2}+(\omega r)^{2}}
$$

Where $\boldsymbol{u}$ is the wind speed, $\omega$ is the angular velocity of the rotor and $\boldsymbol{r}$ is the distance in radial direction from the center of rotation.

The chord size (c) can be calculated using some methodology such as the Blade Element Momentum Theory (BEM), which divides the blade into different sections and in each one, calculates the chord and pitch angle. 
The tip speed ratio TSR is the ratio of the tangential velocity at the blade tip $[\mathrm{m} / \mathrm{s}]$ to the wind speed $\mathrm{u}$ and as a known value is used for the calculation of the fluid velocity $(V)$ and influences the knowledge of the Mach number. The Mach is calculated as the ratio of the maximum $V$ and the speed of sound, as seen in equation (16).

$$
\text { Mach }=\frac{V}{u_{s}}
$$

The angle of attack (AoA) is formed between the fluid velocity vector $(V)$ and the chord line, the performance of the airfoils varies according to this angle and there is a point of maximum performance. This angle of maximum yield is the one used for the calculation of chord size and angle in the BEM theory where $C_{l}$ and $C_{d}$ values are taken into account.

\section{Known values for instance:}

The study considered a TSR equal to 7 and a Reynolds number range between $5 \times 10^{4}$ and $1 \times 10^{5}$. With this TSR the Mach number did not exceed the limit of 0.3 reaching values close to 0.1 , these data were used in the instance for the optimization algorithm.

The initial values for the geometry variables are known, since the shape and characteristics of the SG604X family are taken as their thicknesses.

Other data are known for the purpose of the study to use the final airfoils in a 3-bladed horizontal axis turbine of small size, among these, the diameter of $2 \mathrm{~m}$ and the wind speed for design $4 \mathrm{~m} / \mathrm{s}$.

\section{Results}

As a first step, a selection of the family of airfoils that would be used as a basis for optimization was made. When evaluating the different airfoils, the performance of the SG6043 and SG6040 airfoils stood out Figure 1 shows the selection curves for the airfoil to be used for the mid-blade area, where the SG6043 airfoil was selected. The first selection criterion taken into account was the maximum $\mathrm{C}_{\mathrm{l}} / \mathrm{C}_{\mathrm{d}}$ value where the SG6043 was only surpassed by the BW3, the second evaluation criterion was the slopes of the curves where the BW3 was discarded for having steep slopes which means a greater variation of the performance for different angles of attack (AoA).

For the selection of the airfoil for the blade root, the same evaluation criteria were taken into account as in the selection of the middle-zone blade, the performance of the evaluated airfoils did not have great differences as shown in Figure 2, but the SG6040 was selected because it is a member of the same family than SG6043 airfoil. which means that they have similar shapes.

The SG604X airfoil family is selected, more precisely two profiles are selected, the SG6040 profile designed to be used in the area closest to the blade root and the SG6043 profile designed for the area where more aerodynamics of the blade is needed, which we will call the middle area. Once selected, the Bézier curve parameterization method is used, the shape of the geometry is controlled, as seen in Figure 3, with 13 control points, which form 4 connected curves that turn out to be the shape of the profile.

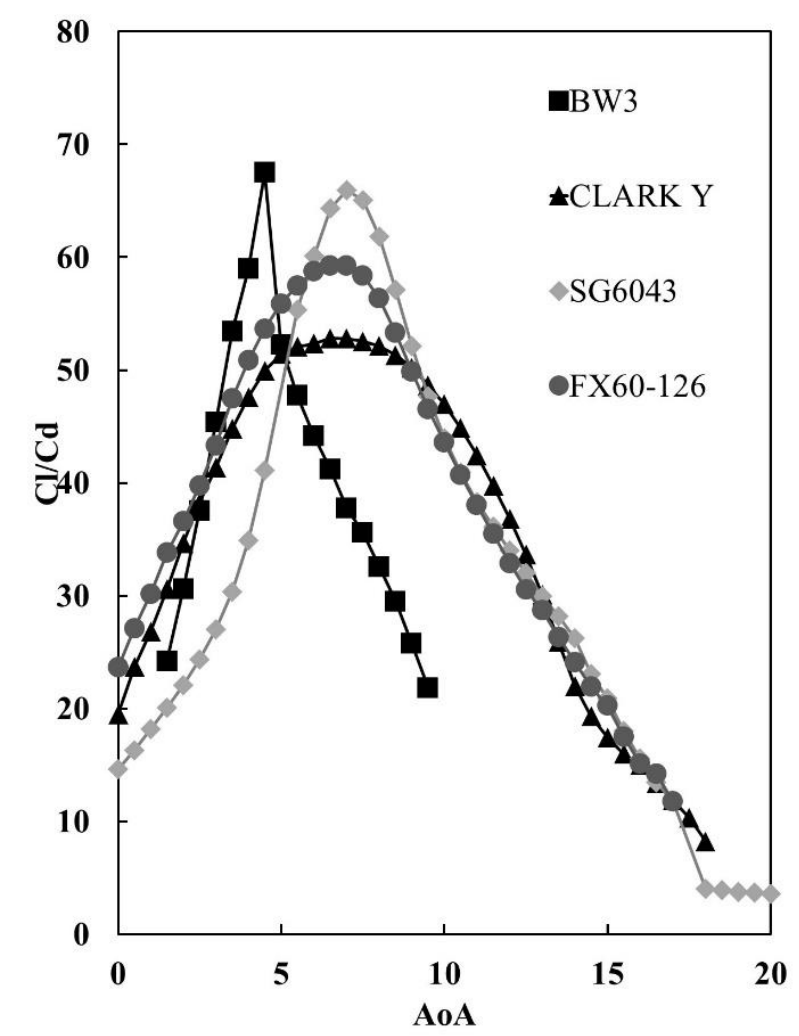

Figure 1 Performance curves comparison at Reynolds number $1 \times 10^{5}$ for the middle zone between multiples airfoils.

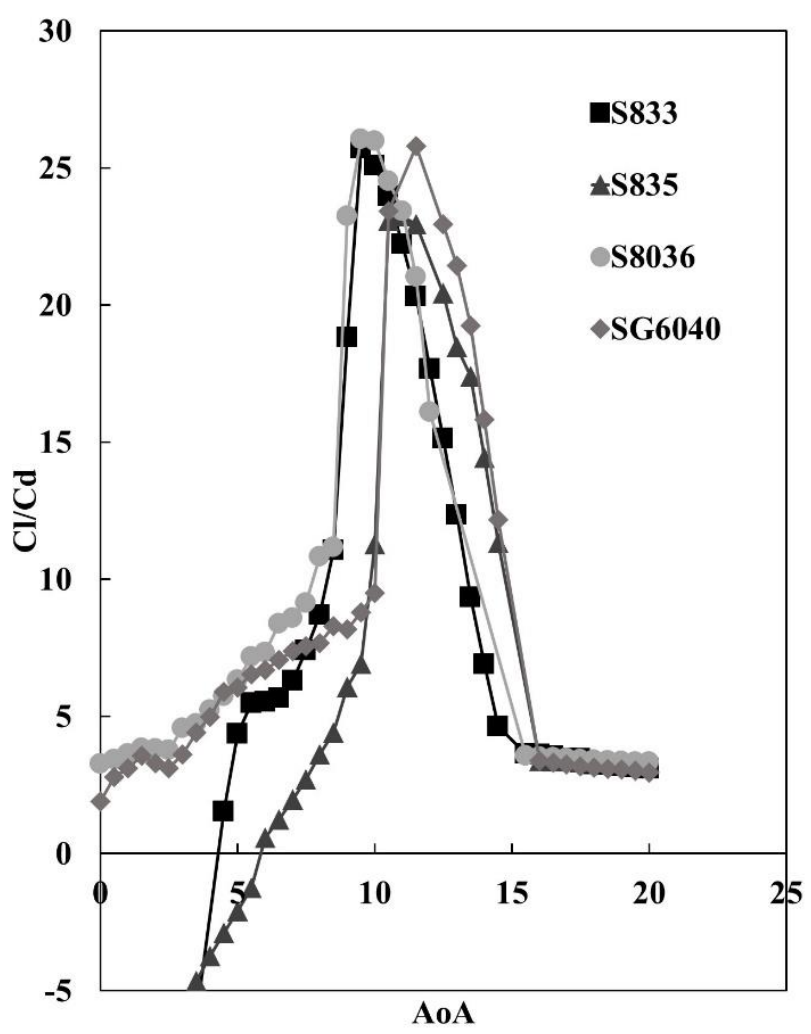

Figure 2 Performance curves comparison at Reynolds number $5 \times 10^{4}$ for the root zone between multiples airfoils. 


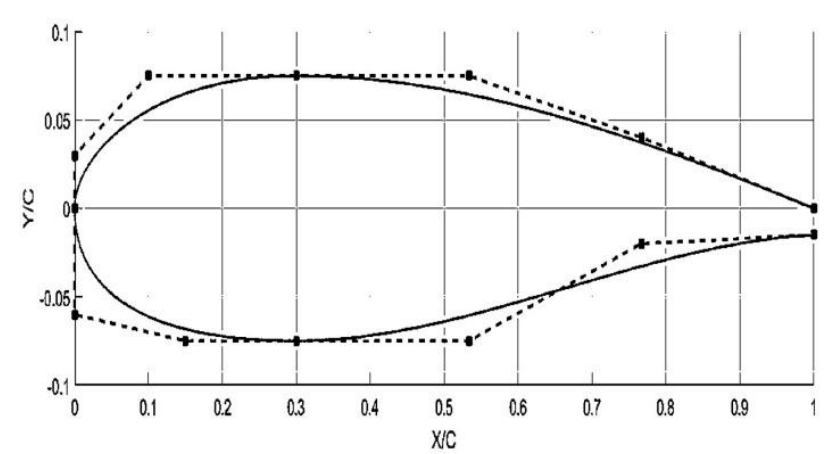

Figure 3. Bézier curves parametrization for airfoil optimization

In the study, during the optimization a new shape is obtained for the airfoil selected for the root, the SG6040. This new airfoil is called RAIZ and in the Figure 4 you can see the curve of the ratio between lift and drag coefficients $\left(C_{l} / C_{d}\right)$ in this graph you can see how the curve of the new airfoil is above the original airfoil and also with less steep slopes. In the same way a new shape called MED is obtained from the airfoil directed to the rest of the blade, the SG6043, In this case the curve of the new shape does not manage to be above that of the original airfoil but it decreases the slope of this curve as can be seen in Figure 5.

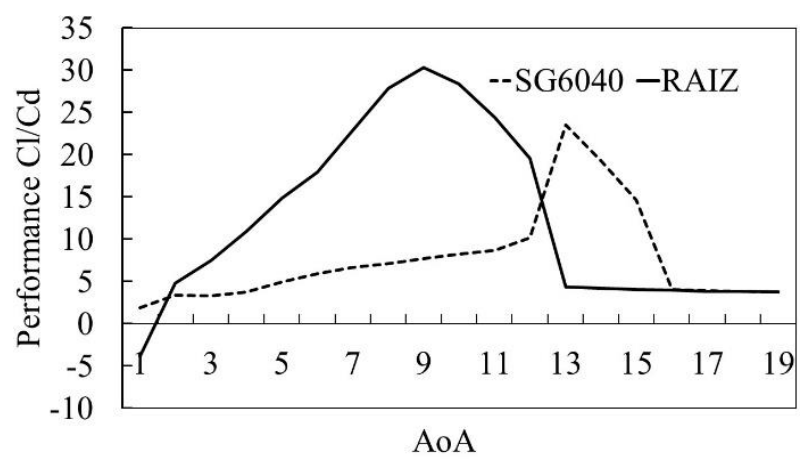

Figure 4. Performance curves comparison at Reynolds number $5 \times 10^{4}$ for the root airfoils between the reference SG6040 and the optimized RAIZ shape.

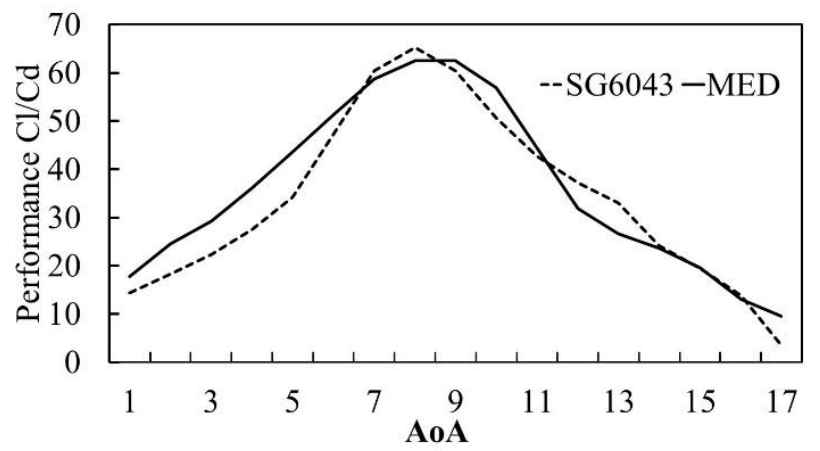

Figure 5. Performance curves comparison at Reynolds number $1 \times 10^{5}$ for the mid zone airfoils between the reference SG6043 and the optimized MED shape.

Once the new shape airfoils are obtained, the next step will be to make a model of the wind turbine blade based on the BEM methodology, with which the chord size and pitch angle will be calculated for each section along the blade.
That results are represented in the Figure 6 and Figure 7, were the is possible to see the variation of the chord between $15 \%$ and $2.8 \%$ of the blade, which is the same as $0.15 \mathrm{~m}$ and $0.028 \mathrm{~m}$. the distribution of the airfoils shape corresponding to $1 / 3$ of the blade length near to the root for RAIZ airfoil and 2/3 remaining for MED airfoil.

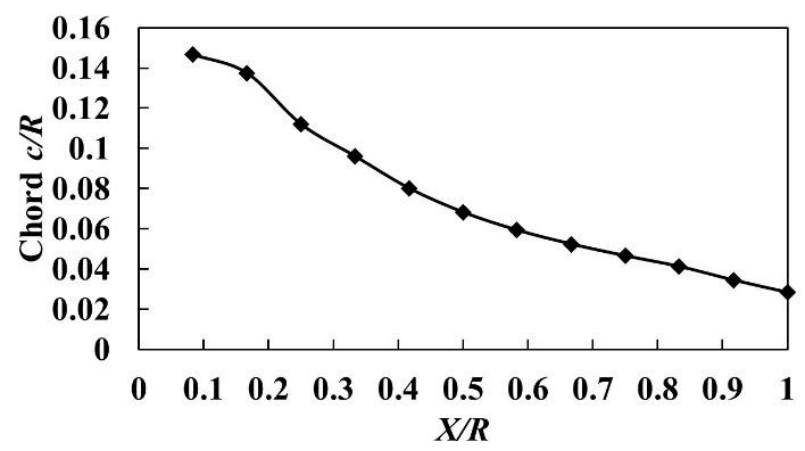

Figure 6 chord distribution of the blade calculated by BEM $(\mathrm{R}=1 \mathrm{~m}) \mathrm{c}$ in meters.

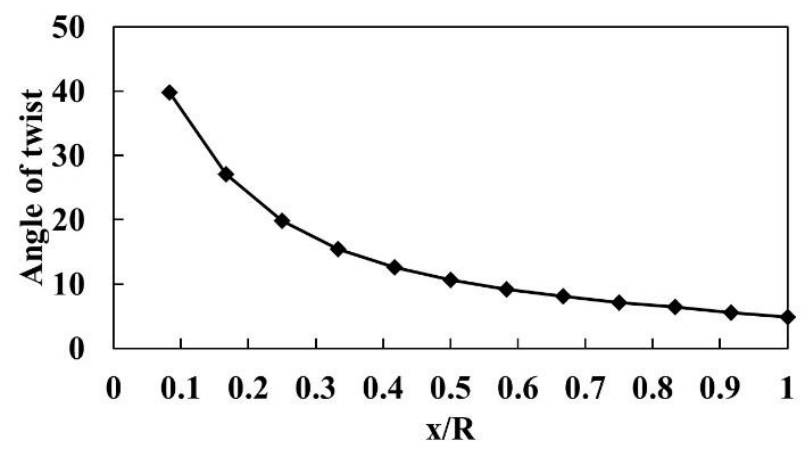

Figure 7 angle of twist distribution along the blade calculated by BEM methodology, $(\mathrm{R}=1 \mathrm{~m})$, angle in degrees.

With the geometrical shape of the blades, a dynamic CFD analysis of the rotor seems to be the next step in this study, although the computational cost is a limitation due to the time it takes for a sufficiently robust simulation. In the first analysis as turbulence model for the solution of the RANS equations is selected the SST $k-\omega$ model and taking into account a $y+$ close to 1.5 , with these parameters is obtained a rotation speed close to $230 \mathrm{rpm}$ with a value of moment of inertia of 0.26 introduced taking into account aluminum as the material selected for the calculation. Studies are still in progress.

\section{Conclusion}

The Gradient based optimization method resulted in an airfoil shape (RAIZ airfoil) for the blade root that has a higher performance than the airfoil used as a basis (SG6040 airfoil) while the selected airfoil for the middle zone SG6043 the new shape called MED does not exceed its maximum value of performance but, the curve has a less abrupt change of the $C_{1} / C_{d}$ ratio for different angles of attack.

Studies on the performance of the model against the fluid and structural analysis of the blades are still needed to define the possible loads to be supported and a suitable material, however, the data obtained so far show a functional model of the blades. 


\section{Acknowledgments}

The authors gratefully acknowledge the financial support provided by the Colombia Scientific Program within the framework of the call Ecosistema Científico (Contract No. FP44842-218-2018).

\section{References}

[1] A. Beltrán-Telles, M. Morera-Hernández, F. E. LópezMonteagudo, and R. Villela-Varela, "Prospectiva de las energías eólica y solar fotovoltaica en la producción de energía eléctrica," CienciaUAT, vol. 11, no. 2, pp. 105117, 2017.

[2] J. F. Ruíz Murcia, J. Serna Cuenca, and H. J. Zapata Lesmes, Atlas de Viento de Colombia. Bogotá D.C. Colombia, 2017.

[3] P. A. C. Rocha et al., "The effects of blade pitch angle on the performance of small-scale wind turbine in urban environments," Energy, vol. 148, pp. 169-178, 2018.

[4] W. C. Wang and H. Y. Teah, "Life cycle assessment of small-scale horizontal axis wind turbines in Taiwan," $J$. Clean. Prod., vol. 141, pp. 492-501, 2017.

[5] N. Karthikeyan, K. K. Murugavel, S. A. Kumar, and S. Rajakumar, "Review of aerodynamic developments on small horizontal axis wind turbine blade," Renew. Sustain. Energy Rev., vol. 42, pp. 801-822, 2015.

[6] E. Basta, M. Ghommem, L. Romdhane, and A. Abdelkefi, "Modeling and experimental comparative analysis on the performance of small-scale wind turbines," Wind Struct., vol. 30, no. 3, pp. 261-273, 2020.

[7] M. Brendel and T. J. Mueller, "Boundary-layer measurements on an airfoil at low Reynolds numbers," J. Aircr., vol. 25, no. 7, pp. 612-617, 1988.

[8] A. Santhanakrishnan et al., "Flow structure and force generation on flapping wings at low reynolds numbers relevant to the flight of tiny insects," Fluids, vol. 3, no. 3, p. 45, 2018.

[9] W.-C. Wang, W. T. Chong, and T.-H. Chao, "Performance analysis of a cross-axis wind turbine from wind tunnel experiments," J. Wind Eng. Ind. Aerodyn., vol. 174, pp. 312-329, 2018.

[10] C. Yan and C. L. Archer, "Assessing compressibility effects on the performance of large horizontal-axis wind turbines," Appl. Energy, vol. 212, pp. 33-45, 2018.

[11] L. Menegozzo, A. Dal Monte, E. Benini, and A. Benato, "Small wind turbines: A numerical study for aerodynamic performance assessment under gust conditions," Renew. energy, vol. 121, pp. 123-132, 2018.

[12] A. C. Benim, M. Diederich, and B. Pfeiffelmann, "Aerodynamic optimization of airfoil profiles for small horizontal axis wind turbines," Computation, vol. 6, no. 2, 2018.

[13] J. Morgado, R. Vizinho, M. A. R. Silvestre, and J. C. Páscoa, "XFOIL vs CFD performance predictions for high lift low Reynolds number airfoils," Aerosp. Sci. Technol., vol. 52, pp. 207-214, 2016.

[14] Y. Liu, C. Yang, and X. Song, "An airfoil parameterization method for the representation and optimization of wind turbine special airfoil," J. Therm. Sci., vol. 24, no. 2, pp. 99-108, 2015.

[15] M. H. S. Abobaker, "Low Reynolds number airfoils," Универзитет у Београду, 2018.

[16] F. Grasso, "Multi-Objective Numerical Optimization applied to Università degli Studi di Napoli ' Federico II
' Doctoral Thesis in Aerospace Engineering MultiObjective Numerical Optimization Applied to Aircraft Design," no. December 2008, 2008.

[17] F. Grasso, "Design of a family of new advanced airfoils for low wind class turbines," J. Phys. Conf. Ser., vol. 555, no. 1, pp. 0-9, 2014. 